\title{
Pseudorandom reliable algorithms and their applications to amphibious communication
}

Weihong Huang, Jian Li

Weihong Huang, Jian Li, "Pseudorandom reliable algorithms and their applications to amphibious communication," Proc. SPIE 7490, PIAGENG 2009: Intelligent Information, Control, and Communication Technology for Agricultural Engineering, 749022 (10 July 2009); doi: 10.1117/12.836811

SDIE Event: International Conference on Photonics and Image in Agriculture Engineering (PIAGENG 2009), 2009, Zhangjiajie, China 


\section{Pseudorandom reliable algorithms and their applications to amphibious communication (Withdrawal Notice)}

Proc. SPIE 7490, 749022 (2009); http://dx.doi.org/10.1117/12.836811

Online Publication Date: 10 July 2009

Withdrawn from Publication: 13 November 2015

Conference Date: 11-12 July 2009

Conference Location: Zhangjiajie, China

Conference Title: PIAGENG 2009: Intelligent Information, Control, and Communication Technology for Agricultural Engineering

Conference Chairs: Honghua Tan, Qi Luo

\section{Weihong Huang}

Hunan Univ. of Science and Technology (China)

\section{Jian Li}

Hunan Univ. (China)

This paper has been identified by SPIE as fraudulent and was withdrawn on 13 November 2015.

PIAGENG 2009: Intelligent Information, Control, and Communication Technology 